\title{
Water Drainage Model
}

\author{
N.D. Rosenberg
}

\section{September 27, 1999}

U.S. Department of Energy

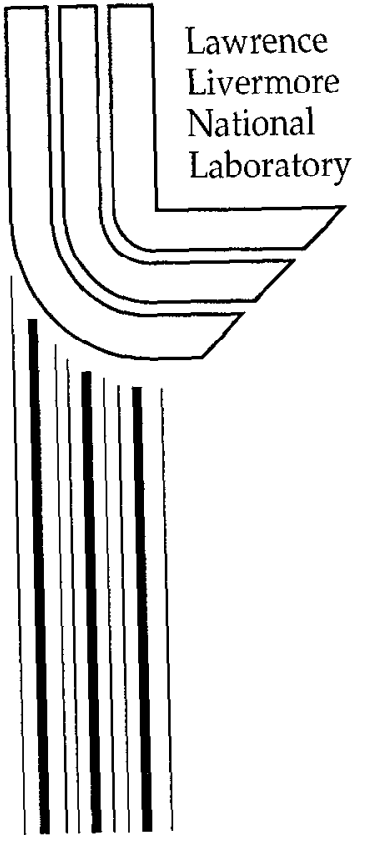




\section{DISCLAIMER}

This document was prepared as an account of work sponsored by an agency of the United States Government. Neither the United States Government nor the University of California nor any of their employees, makes any warranty, express or implied, or assumes any legal liability or responsibility for the accuracy, completeness, or usefulness of any information, apparatus, product, or process disclosed, or represents that its use would not infringe privately owned rights. Reference herein to any specific commercial product, process, or service by trade name, trademark, manufacturer, or otherwise, does not necessarily constitute or imply its endorsement, recommendation, or favoring by the United States Government or the University of California. The views and opinions of authors expressed herein do not necessarily state or reflect those of the United States Government or the University of California, and shall not be used for advertising or product endorsement purposes.

Work performed under the auspices of the U. S. Department of Energy by the University of California Lawrence Livermore National Laboratory under Contract W-7405-Eng-48.

This report has been reproduced directly from the best available copy.

Available to DOE and DOE contractors from the Office of Scientific and Technical Information

P.O. Box 62, Oak Ridge, TN 37831

Prices available from (423) 576-8401

http://apollo.osti.gov/bridge/

Available to the public from the National Technical Information Service

U.S. Department of Commerce 5285 Port Royal Rd., Springfield, VA 22161 http://www.ntis.gov/

OR

Lawrence Livermore National Laboratory

Technical Information Department's Digital Library http://www.llnl.gov/tid/Library.html 


\section{CONTENTS}

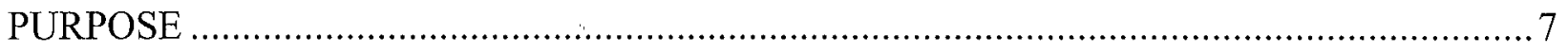

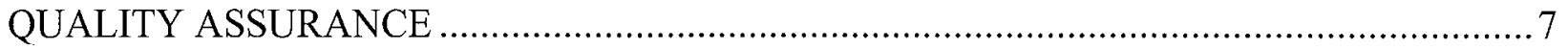

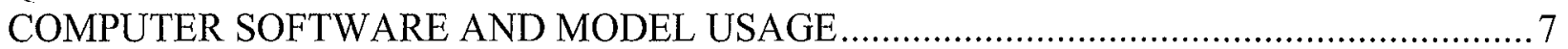

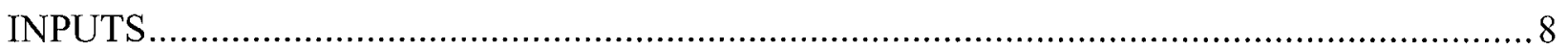

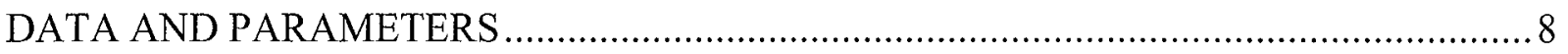

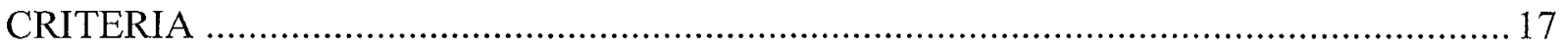

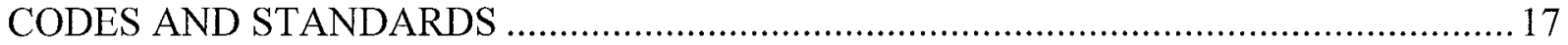

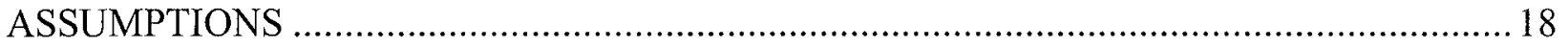

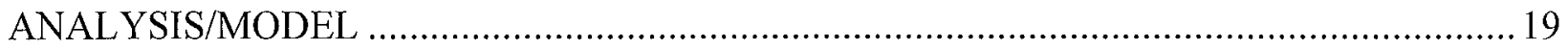

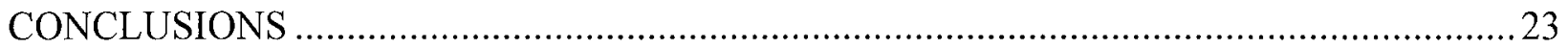

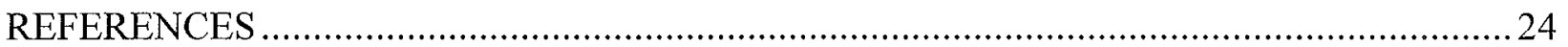

\section{ATTACHMENTS/APPENDICES}

I. DIRS

II. NUFT input files 


\section{TABLES and FIGURES}

Tables

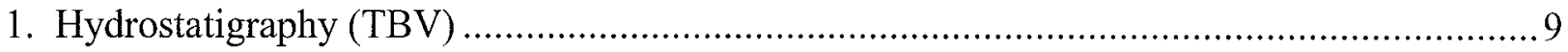

2. Repository Location Relative to Hydrostratigraphy (TBV) ...................................... 10

3. Ground Surface and Water Table Conditions (TBV) .............................................. 10

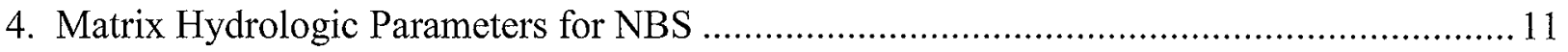

5. Fracture Hydrologic Parameters for NBS ........................................................... 12

6. Hydrologic Parameters for Fracture-Matrrix Interaction for NBS ............................ 13

7. Thermal Parameters and Tortuosity Factor for NBS .................................................... 14

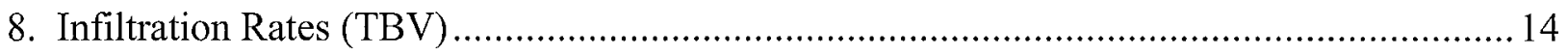

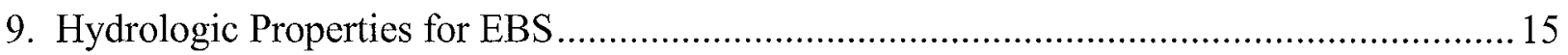

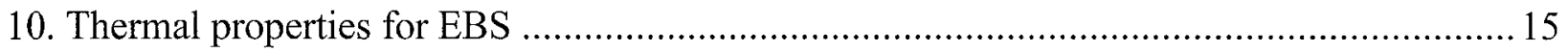

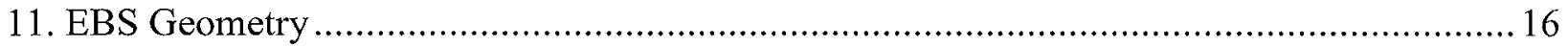

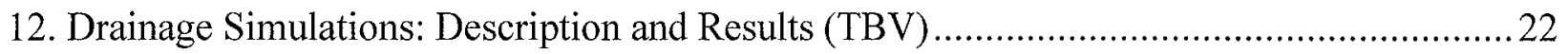

Figures

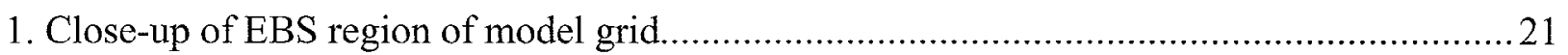




\section{ABBREVIATIONS AND ACRONYMS}

2D two-dimensional

AFC active fracture concept

AMR analysis and modeling report

DKM dual-permeability method

DTN data tracking number

EBS

NBS

NUFT

QA

TBV

engineered barrier system

natural barrier system

nonisothermal unsaturated-saturated flow and transport

quality assurance

$\mathrm{TH}$

to be verified

THC

thermal-hydrolgoic

thermal-hydrological-chemical

THM thermal-hydrological-mechanical

UZ unsaturated zone

WP waste package 


\section{PURPOSE}

The purpose of the water drainage model presented in this AMR is to assess the ability of the EBS to drain water that may enter the EBS.

\section{QUALITY ASSURANCE}

This AMR has been developed in accordance with AP-3.10Q, as an implementing document of Work Package 12012383MX and Development Plan TDP-EBS-MD-000013. An activity evaluation, performed in accordance with QAP-2-0 (EBS Performance Modeling, MOL.19990719.0317), determined that this is a quality-effecting document.

The model developed in this AMR will be validated against appropriate experimental data as data becomes available.

Qualified, Unqualified, and Accepted input data and references have been identified, and TBV assigned and documented in Attachment 1 of this report, in accordance with AP-3.15.

Computer Software and Model Usage are discussed in Section 3 of this report. The following unqualified codes are used: NUFT 3.0s, Software Tracking Number is: 10088-3.0s-00. Model results generated using these codes are identified as TBV. Code validation will be addressed as part of the code qualification effort.

In addition to AP-3.10Q, the following procedures are applicable to this document: AP-6.1Q, AP-3.14Q, AP-3.17Q, AP-SI.1Q, AP-SIII.2Q, YAP.SV.1Q

\section{COMPUTER SOFTWARE AND MODEL USAGE}

The calculations described in this AMR are performed using the USNT module of NUFT v. 3.0s, run on a SUN Ultra 10 workstation. The Software Tracking Number is: $10088-3.0 \mathrm{~s}-00$.

NUFT 3.0s is currently unqualified software due to resource and schedule constraints. Therefore all calculations reported in this AMR must be considered as unqualified (TBV). NUFT 3.0s is in the process of being qualified. The current estimated completion date is 10/1/99. We fully expect that calculation results reported here and the results of future calculations done with the qualified code will be identical. 


\section{INPUTS}

\section{DATA AND PARAMETERS}

\section{NBS}

Table 1 describes a representative column of hydrostratigraphic units at a location at the approximate center of the proposed repository at Yucca Mountain. As of mid-September 1999, this information was not available from the Yucca Mountain technical databases, so all values in Table 1 must be regarded as TBV.

Table 2 gives parameters that described the repository plane with respect to the repository location represented by Table 2. Due to the TBV status of Table 1, this information although based on information from DTN SN9908T0872799.004, must also be considered TBV.

Table 3 lists assumed ground surface temperature and pressure, and water table temperature at the repository location represented by the column in Table 1. Values listed in Table 3 must be regarded at this time as TBV.

Tables 4-7 list hydrologic and thermal properties for the model units listed in Table 1. All these data are from DTN LB997141233129.001. These hydrologic parameters are labeled in this DTN as "1-D only, calibrated flow parameters for a basecase infiltration".

Table 8 lists the infiltration rates that corresponds these parameters. These infiltration rates must be considered TBV.

\section{EBS}

Table 9 and 10 list hydrologic and thermal properties for EBS materials. This information is from Design Input Transmittal PA-SSR-99218.Tb.

Table 11 gives information on the EBS design. This information is from Design Input Transmittal PA-SSR-99218.Tb.

\section{NUFT Input}

The parameters listed above are converted to NUFT input parameters Nitao (1998a, 1998b). Some of the parameters used in NUFT $v 3.0$ s are not described in these reports, will be described in the NUFT v. 3.0s final report which will be available when v. 3.0s is qualified.

Because the UZ Site-Scale Model provides hydrologic data based on an active fracture dual permeability model and the EBS parameters were given based on continuum permeability measurements, we converted the EBS parameters to parameters that would be appropriate for an active fracture dual permeability model. 
Table 1 Hydrostatigraphy (TBV)

\begin{tabular}{|c|c|}
\hline Model Unit & Thickness (m) \\
\hline & Ground surface \\
\hline tcw 11 & 0. \\
\hline tcw12 & 83.086 \\
\hline tcw13 & 5.391 \\
\hline ptn21 & 4.893 \\
\hline ptn22 & 3.193 \\
\hline ptn23 & 2.754 \\
\hline ptn24 & 7.061 \\
\hline ptn25 & 15.410 \\
\hline ptn26 & 14.648 \\
\hline tsw31 & 1.992 \\
\hline tsw32 & 46.348 \\
\hline tsw33 & 87.382 \\
\hline tsw34 & 33.188 \\
\hline tsw35 & 107.370 \\
\hline tsw36 & 31.377 \\
\hline tsw37 & 15.674 \\
\hline tsw38 & 21.006 \\
\hline tsw39 & 2.871 \\
\hline ch1V! & 0. \\
\hline ch2VI & 0 . \\
\hline $\operatorname{ch} 3 \mathrm{VI}$ & 0 . \\
\hline ch4VI & 0. \\
\hline ch5VI & 0. \\
\hline ch1Ze & 14.004 \\
\hline $\operatorname{ch} 2 Z e$ & 16.523 \\
\hline $\operatorname{ch} 3 Z \mathrm{e}$ & 16.523 \\
\hline ch4Ze & 16.523 \\
\hline $\operatorname{ch} 5 \mathrm{Ze}$ & 16.553 \\
\hline ch6 & 18.867 \\
\hline pp4 & 9.932 \\
\hline pp3 & 30.732 \\
\hline pp2 & 16.846 \\
\hline pp1 & 29.619 \\
\hline bf3 & 0. \\
\hline \multirow[t]{2}{*}{ bf2 } & 0. \\
\hline & Water table \\
\hline
\end{tabular}


Table 2 Repository Location Relative to Hydrostratigraphy (TBV)

\begin{tabular}{|l|l|}
\hline repository elevation $(\mathrm{m})$ & 1073.124 \\
\hline host rock unit & tsw35 \\
\hline distance from repository plane to top of water table $(\mathrm{m})$ & 343.131 \\
\hline
\end{tabular}

Table 3 Ground Surface and Water Table Conditions (TBV)

\begin{tabular}{|l|l|}
\hline Temperature at ground surface & $16.544{ }^{\circ} \mathrm{C}$ \\
\hline Pressure at ground surface & $0.851 \mathrm{e} 5 \mathrm{~Pa}$ \\
\hline Air mass fraction at ground surface & 0.986 \\
\hline Temperature at water table & $32.39{ }^{\circ} \mathrm{C}$ \\
\hline Pressure at water table & $0.92 \mathrm{e} 5 \mathrm{~Pa}$ \\
\hline
\end{tabular}


Table 4 Matrix Hydrologic Parameters for NBS

\begin{tabular}{|c|c|c|c|c|c|c|}
\hline \multicolumn{7}{|c|}{ matrix parameters } \\
\hline $\begin{array}{l}\text { Model } \\
\text { Layer }\end{array}$ & permeability $\mathrm{m}^{2}$ & porosity & $\begin{array}{c}\text { Van } \\
\text { Genuchten } \\
\alpha 1 / \mathrm{Pa}\end{array}$ & $\begin{array}{c}\text { Van } \\
\text { Genuchten } \\
\mathrm{m}\end{array}$ & $\begin{array}{c}\text { residual } \\
\text { saturation }\end{array}$ & $\begin{array}{l}\text { satiated } \\
\text { saturation }\end{array}$ \\
\hline tcw11 & $3.86 \mathrm{E}-15$ & 0.253 & $4.00 \mathrm{E}-05$ & 0.47 & 0.07 & 1 \\
\hline tcw12 & $2.74 \mathrm{E}-19$ & 0.082 & 1.81E-05 & 0.241 & 0.19 & 1 \\
\hline tcw13 & $9.23 \mathrm{E}-17$ & 0.203 & $3.44 \mathrm{E}-06$ & 0.398 & 0.31 & 1 \\
\hline ptn21 & $9.90 \mathrm{E}-13$ & 0.387 & $1.01 \mathrm{E}-05$ & 0.176 & 0.23 & 1 \\
\hline ptn22 & $2.65 E-12$ & 0.439 & $1.60 \mathrm{E}-04$ & 0.326 & 0.16 & 1 \\
\hline$\overline{p t n 23}$ & $1.23 E-13$ & 0.254 & $5.58 \mathrm{E}-06$ & 0.397 & 0.08 & 1 \\
\hline ptn24 & $7.86 \mathrm{E}-14$ & 0.411 & $1.53 \mathrm{E}-04$ & 0.225 & 0.14 & 1 \\
\hline ptn25 & $7.00 \mathrm{E}-14$ & 0.499 & $5.27 \mathrm{E}-05$ & 0.323 & 0.06 & 1 \\
\hline ptn26 & $2.21 \mathrm{E}-13$ & 0.492 & $2.49 \mathrm{E}-04$ & 0.285 & 0.05 & 1 \\
\hline tsw31 & $6.32 \mathrm{E}-17$ & 0.053 & $3.61 \mathrm{E}-05$ & 0.303 & 0.22 & 1 \\
\hline tsw32 & $5.83 \mathrm{E}-16$ & 0.157 & $3.61 \mathrm{E}-05$ & 0.333 & 0.07 & 1 \\
\hline tsw33 & $3.08 \mathrm{E}-17$ & 0.154 & $2.13 \mathrm{E}-05$ & 0.298 & 0.12 & 1 \\
\hline tsw34 & $4.07 E-18$ & 0.11 & $3.86 \mathrm{E}-06$ & 0.291 & 0.19 & 1 \\
\hline tsw35 & $3.04 \mathrm{E}-17$ & 0.131 & $6.44 \mathrm{E}-06$ & 0.236 & 0.12 & 1 \\
\hline tsw36 & $5.71 \mathrm{E}-18$ & 0.112 & $3.55 \mathrm{E}-06$ & 0.38 & 0.18 & 1 \\
\hline tsw37 & $4.49 \mathrm{E}-18$ & 0.094 & $5.33 \mathrm{E}-06$ & 0.425 & 0.25 & 1 \\
\hline tsw38 & $4.53 \mathrm{E}-18$ & 0.037 & $6.94 \mathrm{E}-06$ & 0.324 & 0.44 & 1 \\
\hline$\overline{\text { tsw39 }}$ & $5.46 \mathrm{E}-17$ & 0.173 & $2.29 E-05$ & 0.38 & 0.29 & 1 \\
\hline $\operatorname{ch1z}$ & $1.96 \mathrm{E}-19$ & 0.288 & $2.68 \mathrm{E}-07$ & 0.316 & 0.33 & 1 \\
\hline $\operatorname{ch1v}$ & $9.90 \mathrm{E}-13$ & 0.273 & 1.43E-05 & 0.35 & 0.03 & 1 \\
\hline $\operatorname{ch} 2 v$ & $9.27 \mathrm{E}-14$ & 0.345 & $5.13 \mathrm{E}-05$ & 0.299 & 0.07 & 1 \\
\hline $\operatorname{ch} 3 v$ & $9.27 E-14$ & 0.345 & $5.13 E-05$ & 0.299 & 0.07 & 1 \\
\hline $\operatorname{ch} 4 \mathrm{v}$ & $9.27 \mathrm{E}-14$ & 0.345 & $5.13 \mathrm{E}-05$ & 0.299 & 0.07 & 1 \\
\hline $\operatorname{ch} 5 v$ & $9.27 \mathrm{E}-14$ & 0.345 & $5.13 \mathrm{E}-05$ & 0.299 & 0.07 & 1 \\
\hline $\operatorname{ch} 2 z$ & $6.07 E-18$ & 0.331 & $3.47 E-06$ & 0.244 & 0.28 & 1 \\
\hline $\operatorname{ch} 3 z$ & $6.07 E-18$ & 0.331 & $3.47 \mathrm{E}-06$ & 0.244 & 0.28 & 1 \\
\hline $\operatorname{ch} 4 z$ & $6.07 \mathrm{E}-18$ & 0.331 & $3.47 \mathrm{E}-06$ & 0.244 & 0.28 & 1 \\
\hline $\operatorname{ch} 5 z$ & $6.07 \mathrm{E}-18$ & 0.331 & $3.47 \mathrm{E}-06$ & 0.244 & 0.28 & 1 \\
\hline ch6 & $4.23 \mathrm{E}-19$ & 0.266 & $3.38 \mathrm{E}-07$ & 0.51 & 0.37 & 1 \\
\hline$\overline{\mathrm{pp} 4}$ & $4.28 \mathrm{E}-18$ & 0.325 & $1.51 \mathrm{E}-07$ & 0.676 & 0.28 & 1 \\
\hline pp3 & $2.56 \mathrm{E}-14$ & 0.303 & $2.60 \mathrm{E}-05$ & 0.363 & 0.1 & 1 \\
\hline $\mathrm{pp} 2$ & $1.57 \mathrm{E}-16$ & 0.263 & 2.67E-06 & 0.369 & 0.18 & 1 \\
\hline $\mathrm{pp} 1$ & $6.40 \mathrm{E}-17$ & 0.28 & $1.14 \mathrm{E}-06$ & 0.409 & 0.3 & 1 \\
\hline bf3 & $2.34 \mathrm{E}-14$ & 0.115 & $4.48 \mathrm{E}-06$ & 0.481 & 0.11 & 1 \\
\hline $\mathrm{bf} 2$ & $2.51 \mathrm{E}-17$ & 0.259 & $1.54 \mathrm{E}-07$ & 0.569 & 0.18 & 1 \\
\hline
\end{tabular}


Table 5 Fracture Hydrologic Parameters for NBS

\begin{tabular}{|c|c|c|c|c|c|c|}
\hline \multicolumn{7}{|c|}{ fracture parameters } \\
\hline $\begin{array}{l}\text { Model } \\
\text { Layer }\end{array}$ & permeability $\mathrm{m}^{2}$ & porosity & $\begin{array}{c}\text { Van } \\
\text { Genuchten } \\
\alpha 1 / \mathrm{Pa}\end{array}$ & $\begin{array}{c}\text { Van } \\
\text { Genuchten } \\
m\end{array}$ & $\begin{array}{l}\text { residual } \\
\text { saturation }\end{array}$ & $\begin{array}{l}\text { satiated } \\
\text { saturation }\end{array}$ \\
\hline tcw11 & $2.41 \mathrm{E}-12$ & $2.80 \mathrm{E}-02$ & $3.15 E-03$ & 0.627 & 0.01 & 1 \\
\hline tcw12 & $1.00 \mathrm{E}-10$ & $2.00 \mathrm{E}-02$ & $2.13 \mathrm{E}-03$ & 0.613 & 0.01 & 1 \\
\hline tcw13 & $5.42 E-12$ & $1.50 \mathrm{E}-02$ & $1.26 \mathrm{E}-03$ & 0.607 & 0.01 & 1 \\
\hline ptn21 & $1.86 \mathrm{E}-12$ & $1.10 \mathrm{E}-02$ & $1.68 \mathrm{E}-03$ & 0.58 & 0.01 & 1 \\
\hline$p \operatorname{tn} 22$ & $2.00 \mathrm{E}-11$ & $1.20 \mathrm{E}-02$ & $7.68 \mathrm{E}-04$ & 0.58 & 0.01 & 1 \\
\hline ptn23 & $2.60 \mathrm{E}-13$ & $2.50 \mathrm{E}-03$ & $9.23 E-04$ & 0.61 & 0.01 & 1 \\
\hline ptn24 & $4.67 \mathrm{E}-13$ & $1.20 E-02$ & 3.37E-03 & 0.623 & 0.01 & 1 \\
\hline ptn25 & $7.03 \mathrm{E}-13$ & $6.20 \mathrm{E}-03$ & $6.33 E-04$ & 0.644 & 0.01 & 1 \\
\hline ptn26 & $4.44 \mathrm{E}-13$ & $3.60 \mathrm{E}-03$ & 2.79E-04 & 0.552 & 0.01 & $\overline{1}$ \\
\hline tsw31 & $3.21 \mathrm{E}-11$ & $5.50 \mathrm{E}-03$ & $2.49 \mathrm{E}-04$ & 0.566 & 0.01 & 1 \\
\hline tsw32 & $1.26 E-12$ & $9.50 \mathrm{E}-03$ & 1.27E-03 & 0.608 & 0.01 & 1 \\
\hline tsw33 & $5.50 \mathrm{E}-13$ & $6.60 \mathrm{E}-03$ & $1.46 \mathrm{E}-03$ & 0.608 & 0.01 & 1 \\
\hline tsw34 & $2.76 \mathrm{E}-13$ & $1.00 \mathrm{E}-02$ & $5.16 \mathrm{E}-04$ & 0.608 & 0.01 & 1 \\
\hline tsw35 & $1.29 \mathrm{E}-12$ & 1.10E-02 & 7.39E-04 & 0.611 & 0.01 & 1 \\
\hline tsw36 & $9.91 \mathrm{E}-13$ & $1.50 \mathrm{E}-02$ & 7.84E-04 & 0.61 & 0.01 & 1 \\
\hline tsw37 & $9.91 \mathrm{E}-13$ & $1.50 \mathrm{E}-02$ & $7.84 \mathrm{E}-04$ & 0.61 & 0.01 & 1 \\
\hline tsw38 & $5.92 \mathrm{E}-13$ & $1.20 \mathrm{E}-02$ & $4.87 \mathrm{E}-04$ & 0.612 & 0.01 & 1 \\
\hline tsw39 & $4.57 \mathrm{E}-13$ & $4.60 \mathrm{E}-03$ & $9.63 E-04$ & 0.634 & 0.01 & 1 \\
\hline $\operatorname{ch} 1 z$ & $3.40 \mathrm{E}-13$ & $1.70 \mathrm{E}-04$ & $1.43 E-03$ & 0.631 & 0.01 & 1 \\
\hline $\operatorname{ch} 1 v$ & $1.84 \mathrm{E}-12$ & $6.90 \mathrm{E}-04$ & $1.09 \mathrm{E}-03$ & 0.624 & 0.01 & 1 \\
\hline $\operatorname{ch} 2 v$ & $2.89 \mathrm{E}-13$ & 8.90E-04 & $5.18 E-04$ & 0.628 & 0.01 & 1 \\
\hline $\operatorname{ch} 3 v$ & $2.89 \mathrm{E}-13$ & $8.90 E-04$ & $5.18 \mathrm{E}-04$ & 0.628 & 0.01 & 1 \\
\hline $\operatorname{ch} 4 v$ & $2.89 \mathrm{E}-13$ & $8.90 E-04$ & $5.18 \mathrm{E}-04$ & 0.628 & 0.01 & 1 \\
\hline $\operatorname{ch} 5 v$ & $2.89 \mathrm{E}-13$ & 8.90E-04 & $5.18 \mathrm{E}-04$ & 0.628 & 0.01 & 1 \\
\hline ch2z & $3.12 E-14$ & $4.30 \mathrm{E}-04$ & $4.88 E-04$ & 0.598 & 0.01 & 1 \\
\hline $\operatorname{ch} 3 z$ & $3.12 \mathrm{E}-14$ & 4.30E-04 & $4.88 E-04$ & 0.598 & 0.01 & 1 \\
\hline ch4z & $3.12 \mathrm{E}-14$ & $4.30 E-04$ & $4.88 E-04$ & 0.598 & 0.01 & 1 \\
\hline ch5z & $3.12 E-14$ & $4.30 \mathrm{E}-04$ & $4.88 \mathrm{E}-04$ & 0.598 & 0.01 & 1 \\
\hline ch6 & $1.67 \mathrm{E}-14$ & $1.70 \mathrm{E}-04$ & $7.49 E-04$ & 0.604 & 0.01 & 1 \\
\hline pp4 & $3.84 \mathrm{E}-14$ & 4.30E-04 & $5.72 E-04$ & 0.627 & 0.01 & 1 \\
\hline pp3 & $7.60 \mathrm{E}-12$ & 1.10E-03 & $8.73 \mathrm{E}-04$ & 0.655 & 0.01 & 1 \\
\hline $\mathrm{pp} 2$ & $1.38 \mathrm{E}-13$ & $1.10 \mathrm{E}-03$ & $1.21 \mathrm{E}-03$ & 0.606 & 0.01 & 1 \\
\hline $\mathrm{pp} 1$ & $1.12 \mathrm{E}-13$ & $4.30 \mathrm{E}-04$ & $5.33 \mathrm{E}-04$ & 0.622 & 0.01 & 1 \\
\hline bf3 & $4.08 \mathrm{E}-13$ & $1.10 \mathrm{E}-03$ & $9.95 \mathrm{E}-04$ & 0.624 & 0.01 & 1 \\
\hline bf2 & $1.30 \mathrm{E}-14$ & $4.30 \mathrm{E}-04$ & $5.42 \mathrm{E}-04$ & 0.608 & 0.01 & 1 \\
\hline
\end{tabular}


Table 6 Hydrologic Parameters for Fracture-Matrrix Interaction for NBS

\begin{tabular}{|c|c|c|c|}
\hline \multicolumn{4}{|c|}{ fracture parameters } \\
\hline Model Layer & $\begin{array}{l}\text { active fracture } \\
\text { parameter }\end{array}$ & $\begin{array}{c}\text { frequency } \\
1 / \mathrm{m}\end{array}$ & $\begin{array}{c}\text { Fracture to matrix } \\
\text { connection area } \\
\mathrm{m}^{2} / \mathrm{m}^{3}\end{array}$ \\
\hline tcw11 & 0.3 & 0.92 & 1.56 \\
\hline tcw12 & 0.3 & 1.91 & 13.39 \\
\hline tcw13 & 0.3 & 2.79 & 3.77 \\
\hline ptn21 & 0.09 & 0.67 & 1 \\
\hline ptn22 & 0.09 & 0.46 & 1.41 \\
\hline ptn23 & 0.09 & 0.57 & 1.75 \\
\hline ptn24 & 0.09 & 0.46 & 0.34 \\
\hline ptn25 & 0.09 & 0.52 & 1.09 \\
\hline ptn26 & 0.09 & 0.97 & 3.56 \\
\hline tsw31 & 0.06 & 2.17 & 3.86 \\
\hline tsw32 & 0.41 & 1.12 & 3.21 \\
\hline tsw33 & 0.41 & 0.81 & 4.44 \\
\hline tsw34 & 0.41 & 4.32 & 13.54 \\
\hline tsw35 & 0.41 & 3.16 & 9.68 \\
\hline tsw36 & 0.41 & 4.02 & 12.31 \\
\hline tsw37 & 0.41 & 4.02 & 12.31 \\
\hline tsw38 & 0.41 & 4.36 & 13.34 \\
\hline tsw39 & 0.41 & 0.96 & 2.95 \\
\hline $\operatorname{ch} 1 z$ & 0.1 & 0.04 & 0.11 \\
\hline $\operatorname{ch} 1 v$ & 0.13 & 0.1 & 0.3 \\
\hline $\operatorname{ch} 2 v$ & 0.13 & 0.14 & 0.43 \\
\hline $\operatorname{ch} 3 v$ & 0.13 & 0.14 & 0.43 \\
\hline $\operatorname{ch} 4 \mathrm{v}$ & 0.13 & 0.14 & 0.43 \\
\hline $\operatorname{ch} 5 \mathrm{v}$ & 0.13 & 0.14 & 0.43 \\
\hline $\operatorname{ch} 2 z$ & 0.1 & 0.14 & 0.43 \\
\hline $\operatorname{ch} 3 z$ & 0.1 & 0.14 & 0.43 \\
\hline $\operatorname{ch} 4 \mathrm{z}$ & 0.1 & 0.14 & 0.43 \\
\hline $\operatorname{ch} 5 z$ & 0.1 & 0.14 & 0.43 \\
\hline ch6 & 0.1 & 0.04 & 0.11 \\
\hline pp4 & 0.1 & 0.14 & 0.43 \\
\hline pp3 & 0.46 & 0.2 & 0.61 \\
\hline $\mathrm{pp} 2$ & 0.46 & 0.2 & 0.61 \\
\hline $\mathrm{pp} 1$ & 0.1 & 0.14 & 0.43 \\
\hline bf3 & 0.46 & 0.2 & 0.61 \\
\hline
\end{tabular}


Table 7 Thermal Parameters and Tortuosity Factor for NBS

\begin{tabular}{|c|c|c|c|c|c|}
\hline Model Layer & $\begin{array}{c}\text { rock grain } \\
\text { density } \\
\mathrm{kg} / \mathrm{m}^{3}\end{array}$ & $\begin{array}{c}\text { rock grain } \\
\text { specific heat } \\
\mathrm{J} / \mathrm{kg} \mathrm{K}\end{array}$ & $\begin{array}{c}\text { dry } \\
\text { conductivity } \\
\text { W/m K }\end{array}$ & $\begin{array}{c}\text { wet } \\
\text { conductivity } \\
\text { W/m K }\end{array}$ & Tortuosity \\
\hline tcw11 & 2550 & 823 & 1.60 & 2.00 & 0.7 \\
\hline tcw12 & 2510 & 851 & 1.24 & 1.81 & 0.7 \\
\hline tcw13 & 2470 & 857 & 0.54 & 0.98 & 0.7 \\
\hline ptn21 & 2380 & 1040 & 0.50 & 1.07 & 0.7 \\
\hline ptn22 & 2340 & 1080 & 0.35 & 0.50 & 0.7 \\
\hline ptn23 & 2400 & 849 & 0.44 & 0.97 & 0.7 \\
\hline$p \operatorname{tn} 24$ & 2370 & 1020 & 0.46 & 1.02 & 0.7 \\
\hline ptn25 & 2260 & 1330 & 0.35 & 0.82 & 0.7 \\
\hline ptn26 & 2370 & 1220 & 0.23 & 0.67 & 0.7 \\
\hline tsw31 & 2510 & 834 & 0.37 & 1.00 & 0.7 \\
\hline tsw32 & 2550 & 866 & 1.06 & 1.62 & 0.7 \\
\hline tsw33 & 2510 & 882 & 0.79 & 1.68 & 0.7 \\
\hline tsw34 & 2530 & 948 & 1.56 & 2.33 & 0.7 \\
\hline tsw35 & 2540 & 900 & 1.20 & 2.02 & 0.7 \\
\hline tsw36 & 2560 & 865 & 1.42 & 1.84 & 0.7 \\
\hline tsw37 & 2560 & 865 & 1.42 & 1.84 & 0.7 \\
\hline tsw38 & 2360 & 984 & 1.69 & 2.08 & 0.7 \\
\hline tsw39 & 2360 & 984 & 1.69 & 2.08 & 0.7 \\
\hline $\operatorname{ch} 1 z$ & 2310 & 1060 & 0.70 & 1.31 & 0.7 \\
\hline $\operatorname{ch} 1 v$ & 2310 & 1060 & 0.70 & 1.31 & 0.7 \\
\hline ch2v & 2240 & 1200 & 0.58 & 1.17 & 0.7 \\
\hline $\operatorname{ch} 3 v$ & 2240 & 1200 & 0.58 & 1.17 & 0.7 \\
\hline $\operatorname{ch} 4 v$ & 2240 & 1200 & 0.58 & 1.17 & 0.7 \\
\hline $\operatorname{ch} 5 v$ & 2240 & 1200 & 0.58 & 1.17 & 0.7 \\
\hline $\operatorname{ch} 2 z$ & 2350 & 1150 & 0.61 & 1.20 & 0.7 \\
\hline $\operatorname{ch} 3 z$ & 2350 & 1150 & 0.61 & 1.20 & 0.7 \\
\hline ch4z & 2350 & 1150 & 0.61 & 1.20 & 0.7 \\
\hline ch5z & 2350 & 1150 & 0.61 & 1.20 & 0.7 \\
\hline ch6 & 2440 & 1170 & 0.73 & 1.35 & 0.7 \\
\hline pp4 & 2410 & 577 & 0.62 & 1.21 & 0.7 \\
\hline pp3 & 2580 & 841 & 0.66 & 1.26 & 0.7 \\
\hline pp2 & 2580 & 841 & 0.66 & 1.26 & 0.7 \\
\hline pp1 & 2470 & 635 & 0.72 & 1.33 & 0.7 \\
\hline bf 3 & 2570 & 763 & 1.41 & 1.83 & 0.7 \\
\hline bf2 & 2410 & 633 & 0.74 & 1.36 & 0.7 \\
\hline
\end{tabular}

Table 8 Infiltration Rates (TBV)

\begin{tabular}{|l|l|l|}
\hline Current Climate & Monsoon & Glacial \\
\hline $10.14 \mathrm{~mm} / \mathrm{yr}$ & $24.09 \mathrm{~mm} / \mathrm{yr}$ & $38.66 \mathrm{~mm} / \mathrm{yr}$ \\
\hline
\end{tabular}


Table 9 Hydrologic Properties for EBS

\begin{tabular}{|c|c|c|c|c|c|c|}
\hline Material & permeability $\mathrm{m}^{2}$ & porosity & $\begin{array}{c}\text { Van } \\
\text { Genuchten } \\
\alpha 1 / \mathrm{Pa}\end{array}$ & $\begin{array}{c}\text { Van } \\
\text { Genuchten } \\
\mathrm{n}\end{array}$ & $\begin{array}{c}\text { residual } \\
\text { saturation }\end{array}$ & $\begin{array}{l}\text { satiated } \\
\text { saturation }\end{array}$ \\
\hline backfill & $1.43 \times 10^{-11}$ & 0.41 & $2.7523 \times 10^{-4}$ & 2.0 & 0.024 & 1 \\
\hline invert & $6.152 \times 10^{-10}$ & 0.545 & $1.2232 \times 10^{-3}$ & 2.7 & 0.092 & 1 \\
\hline
\end{tabular}

Table 10 Thermal properties for EBS

\begin{tabular}{|c|c|c|c|c|c|}
\hline Material & $\begin{array}{c}\text { rock grain } \\
\text { density } \\
\mathrm{kg} / \mathrm{m}^{3}\end{array}$ & $\begin{array}{c}\text { rock grain } \\
\text { specific heat } \\
\mathrm{J} / \mathrm{kg} \mathrm{K}\end{array}$ & $\begin{array}{c}\text { dry } \\
\text { conductivity } \\
\text { W/m K }\end{array}$ & $\begin{array}{c}\text { wet } \\
\text { conductivity } \\
\text { W/m K }\end{array}$ & Tortuosity \\
\hline backfill & 2700 & 795.492 & 0.33 & 0.33 & 0.7 \\
\hline invert & 2530 & 948 & 0.66 & 0.66 & 0.7 \\
\hline
\end{tabular}


Table 11 EBS Geometry

\begin{tabular}{|c|c|c|}
\hline & Value & Source \\
\hline Drift diameter & $5.5 \mathrm{~m}$ & PA-SSR-99218.Tb \\
\hline Waste package outer diameter & $1.67 \mathrm{~m}$ & $\begin{array}{l}\text { Calculated using PA-WP- } \\
\text { 99184.T }\end{array}$ \\
\hline $\begin{array}{l}\text { Location of waste package center above } \\
\text { bottom of drift }\end{array}$ & $1.945 \mathrm{~m}$ & PA-SSR-99218.Tb \\
\hline $\begin{array}{l}\text { Location of waste package center below the } \\
\text { springline }\end{array}$ & $0.805 \mathrm{~m}$ & PA-SSR-99218.Tb \\
\hline Angle of Repose & $26^{\circ}$ & PA-SSR-99218.Tb \\
\hline $\begin{array}{l}\text { Minimum depth of backfill cover (this occurs at } \\
\text { an angle equivalent to the angle of repose } \\
\text { measured off the vertical drawn from the } \\
\text { waste package centerline) }\end{array}$ & $1.495 \mathrm{~m}$ & PA-SSR-99218.Tb \\
\hline Drip shield thickness & $0.02 \mathrm{~m}$ & $\begin{array}{l}\text { B00000000-01717-0210- } \\
\text { 00074 Rev00 } \\
\text { (same as SSR-WP-99242.T) }\end{array}$ \\
\hline $\begin{array}{l}\text { Air gap between waste package surface and } \\
\text { the inside of drip shield }\end{array}$ & $0.396 \mathrm{~m}$ & $\begin{array}{l}\text { Calculated using PA-SSR- } \\
\text { 99218.Tb and waste package } \\
\text { outer diameter above }\end{array}$ \\
\hline $\begin{array}{l}\text { Location of backfill spoil peak (this is the } \\
\text { location where the top of the backfill intersects } \\
\text { the vertical drawn from the drift centerline) } \\
\text { above the drift springline }\end{array}$ & $2.25 \mathrm{~m}$ & PA-SSR-99218.Tb \\
\hline Backfill/drift wall intersection point & $\begin{array}{l}1.0 \mathrm{~m} \text { above } \\
\text { the springline } \\
\text { at the drift } \\
\text { wall } \\
\text { intersection }\end{array}$ & PA-SSR-99218.Tb \\
\hline $\begin{array}{l}\text { Air gap above invert and below waste } \\
\text { package surface }\end{array}$ & $0.504 \mathrm{~m}$ & $\begin{array}{l}\text { Calculated using PA-SSR- } \\
\text { 99218.Tb and waste package } \\
\text { outer diameter above }\end{array}$ \\
\hline Inside radius of drip shield & $1.231 \mathrm{~m}$ & PA-SSR-99218.Tb \\
\hline Top of invert as measured from bottom of drift & $0.606 \mathrm{~m}$ & PA-SSR-99218.Tb \\
\hline Waste package spacing & 0.1 & PA-WP-99184.T \\
\hline Emplacement drift spacing & $81 \mathrm{~m}$ & PA-SSR-99218.Tb \\
\hline
\end{tabular}




\section{CRITERIA}

The model developed in this report is based on a flow through porous media model (unsaturated, nonisothermal), using a dual permeability approach (DKM) with active fractures concept (AFC). This conceptual is consistent with the model approach used in most recent UZ Site-Scale Model. All the simulations presented in this section were performed using the USNT module of NUFT 3.0s. NUFT has been used extensively to simulate thermal-hydrological behavior on the Yucca Mountain Project. For example, NUFT, using the dual permeability approach, but not with the active fracture concept, was the basis for simulating WP environment conditions for the most recent total system performance assessment-viability assessment (OCRWM 1998, Volume 3, Section 3.2. Comparison of models based on this approach against data from the Drift Scale Test are being performed as part of the Thermal Testing AMR. There are no currently available appropriate field or laboratory tests to compare with the drainage calculations presented here, but to the extent that testing is conducted in the future, this model can be compared with those data.

\section{CODES AND STANDARDS}

The model/analysis documented in this report were developed using the applicable QA procedures, and conventional cngincering/scientific standards. 


\section{ASSUMPTIONS}

1. Nonisothermal, unsaturated flow through porous media, using an implicit dual permeability (DKM) with active fracture concept (AFC) as represented by the USNT module of the NUFT 3.0s computer code applies.

2. The UZ Site-Scale Model is representative of the unsaturated hydrology (e.g., hydrologic parameters and unit definition) of the site.

3. A 2-D, steady-state analysis is conservative.

4. The location modeled, approximately in the center of the repository, is representative of the site.

5. If large amounts of water are entering the EBS, then the temperatures in the EBS are below boiling and to first order, the heat given off by waste packages can be neglected for assessing drainage.

6. The key effect of THC/THM or construction processes as they apply to water drainage is to assume that fracture permeability below the drift is significantly decreased. 


\section{ANALYSIS/MODEL}

\section{Methodology}

General - An effort was made to be as consistent as possible with that of other AMRs being prepared for the EBS and NFE PMRs, and the YMP UZ Site-Scale Model.

Conceptual Model - The system is described as steady-state flow through porous media model, using a dual permeability with active fractures approach. Infiltration is applied to the top of the model domain, which represents the ground surface. The region between the top of the drift and the backfill is represented by the host-rock unit so that infiltration applied to the top of the model emters the drift. This is equivalent to applying seepage directly on the top of the backfill, but it preserves the model domain for easier comparison with simulations being performed for other AMRs.

Numerical Model - All the simulations presented in this section were performed using the USNT module of NUFT $3.0 \mathrm{~s}$.

Model Domain - The 2D model domain extends vertically from the ground surface to the water table and contains the model units described in Table 1, and horizontally from the center of a drift to the center of the pillar between the drifts. The grid is rectangular with irregular spacing and contains 1200 cells. The current "chimney" domain model was chosen for consistency with other past and concurrent NUFT EBS modeling efforts and because the boundary conditions are better defined. A close-up of the EBS region of the modeling domain is shown in Figure 1. At the left hand side of this model domain, which represents the center of the EBS, invert is represented by 2 cells.

Hydrologic properties - An active fracture, dual permeability approach was used to represent hydrologic properties. See Tables 4-7. When these values are changed to examine parameter sensitivity, this is explicitly stated. Since the key to drainage is the permeability of the host rock unit below the EBS, we investigate the consequence of changes to the permeability of this rock, in several simulations. Such permeability changes occur due THC/THM or construction processes. In these simulations, the permeability of the fractures in host rock unit directly (1 cell) below the invert was reduced by many orders of magnitude, to the permeability of the host rock matrix. In other cases, the fracture permeability in the host rock directly (1 cell) below the entire EBS was reduced to this value. These permeability reductions will be referred to as "fractures plugged below invert" and "fractures plugged below EBS", respectively. The region immediately above the invert is represented in this model by impermeable material, so ponding of water above the invert can not occur (i.e., water is diverted horizontally).

Boundary Conditions - Boundary conditions for temperature, pressure and relative humidity must be defined. The temperature and pressure parameter values for ground surface and water table listed in Table 3 are held constant. The liquid saturations at the ground surface and water table are held to 0 and 1, respectively, and the air at the ground surface is assumed to have $100 \%$ 
relative humidity. Side boundaries are assumed to be reflective, that is, there is no flow of water, air or heat through the side boundaries.

Infiltration - A constant infiltration is applied to the ground surface. For some simulations, we assumed that infiltration was equal to the current climate or glacial infiltration rate (see Table 3 ). In other simulations, we calculated the infiltration assuming that the infiltration is concentrated spatially such that the entire flux between adjacent pillar centerlines is focused into the intervening drift (i.e., multiply infiltration rate by 40.5/2.75), and then applied that rate across the entire model domain. These rates will be referred as focused infiltration rates. The greatest infiltration rate considered is the focused glacial rate $(38.66 \mathrm{~mm} / \mathrm{yr} \times 40.5 / 2.75=570 \mathrm{~mm} / \mathrm{yr})$.

Performance Goals - The EBS is considered to fail with respect to its ability to drain incoming water when the saturation averaged over the bottom half of the invert at the point furthest from the center of the drift reaches a value of 1 . In the numerical model, this location is represented by a $0.303 \mathrm{~m}$ long by $0.35 \mathrm{~m}$ wide cell. Of the six cells used to represent the invert in the numerical model, this cell was always the most saturated one.

Simulations - A description of the simulations run for this AMR and results of these simulations are given in Table 12. Results are reported in terms of liquid saturation for the cell corresponding to Cell \#1 marked in Fig. 1. Results are also reported for the cell directly above this cell, marked as Cell \#2 in Fig. 1. Due to the TBV status of the NUFT 3.0s code and much of the data used as model input, these results are marked TBV. 


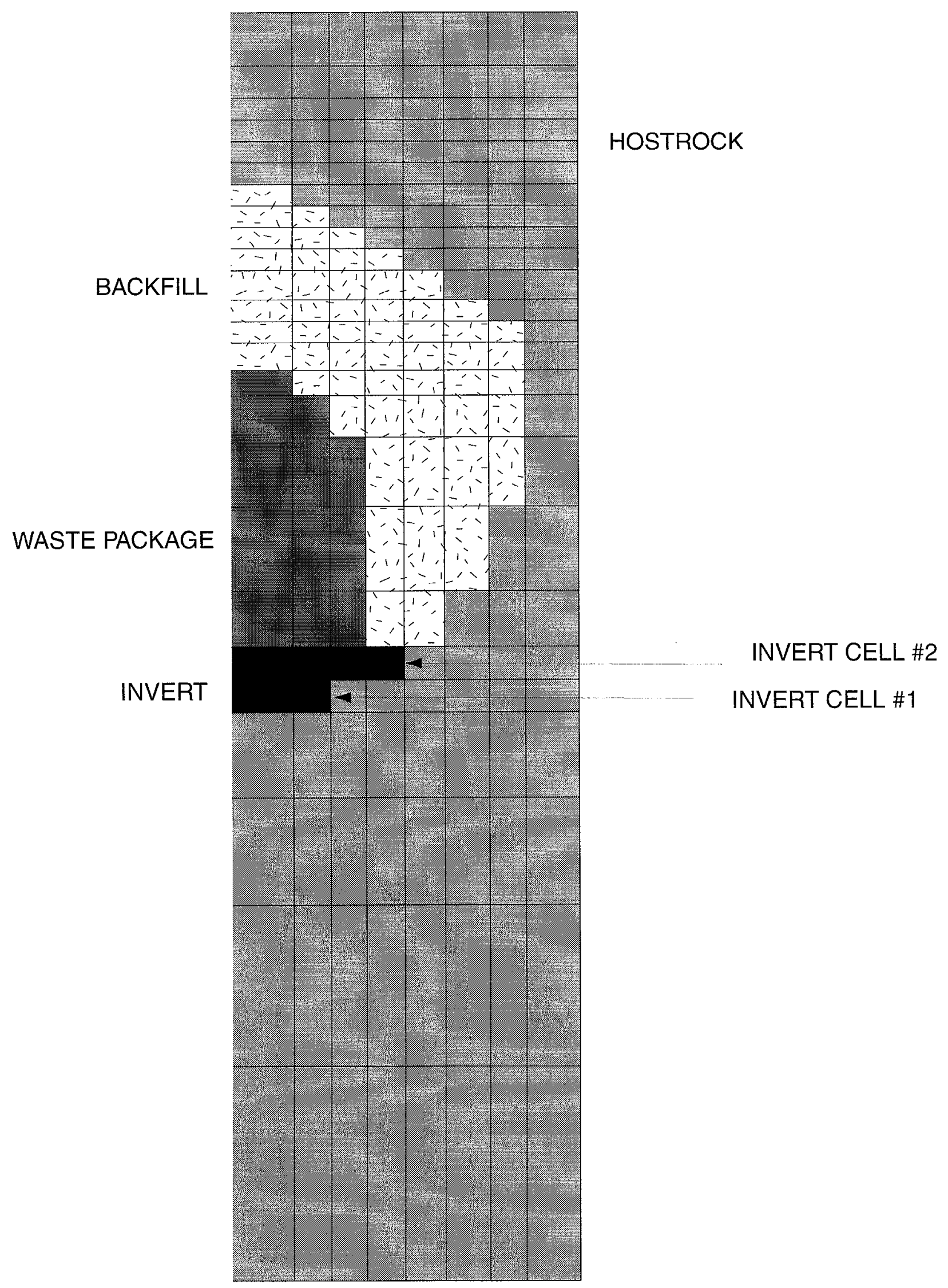

Fig. 1. Close-up of EBS region of model grid 
Table 12 Drainage Simulations: Description and Results (TBV)

\begin{tabular}{|c|c|c|c|c|c|c|}
\hline & \multicolumn{2}{|c|}{ Infiltration Rate } & \multirow{2}{*}{$\begin{array}{c}\text { Changes to EBS } \\
\text { properties }\end{array}$} & \multirow{2}{*}{$\begin{array}{c}\text { Changes to NBS } \\
\text { properties }\end{array}$} & \multirow{2}{*}{$\begin{array}{c}\text { Saturation } \\
\text { Cell \#1 (see } \\
\text { Fig. 1) at } \\
\text { steady-state* } \\
0.189\end{array}$} & \multirow{2}{*}{$\begin{array}{c}\text { Saturation } \\
\text { Cell \#2 (see } \\
\text { Fig. 1) at } \\
\text { steady-state } \\
0.140\end{array}$} \\
\hline$A$ & Glacial & $38.66 \mathrm{~mm} / \mathrm{yr}$ & & & & \\
\hline$B$ & $\begin{array}{l}\text { Focused } \\
\text { Glacial }\end{array}$ & $570 \mathrm{~mm} / \mathrm{yr}$ & & & 0.317 & 0.200 \\
\hline $\mathrm{C}$ & $\begin{array}{l}\text { Focused } \\
\text { Glacial }\end{array}$ & $570 \mathrm{~mm} / \mathrm{yr}$ & $\begin{array}{l}\text { Decrease } \\
\text { backfill } \\
\text { permeability by } \\
10 x\end{array}$ & & 0.299 & 0.176 \\
\hline $\bar{D}$ & $\begin{array}{l}\text { Focused } \\
\text { Glacial }\end{array}$ & $570 \mathrm{~mm} / \mathrm{yr}$ & $\begin{array}{l}\text { Decrease invert } \\
\text { permeability by } \\
10 x\end{array}$ & & 0.317 & 0.20 \\
\hline$E$ & $\begin{array}{l}\text { Focused } \\
\text { Glacial }\end{array}$ & $570 \mathrm{~mm} / \mathrm{yr}$ & $\begin{array}{l}\text { Decrease invert } \\
\text { and backfill } \\
\text { permeability by } \\
10 x\end{array}$ & & 0.299 & 0.176 \\
\hline$F$ & Glacial & $38.66 \mathrm{~mm} / \mathrm{yr}$ & & $\begin{array}{l}\text { fractures } \\
\text { plugged below } \\
\text { invert }\end{array}$ & 0.756 & 0.163 \\
\hline G & 2xGlacial & $77 \mathrm{~mm} / \mathrm{yr}$ & & $\begin{array}{l}\text { fractures } \\
\text { plugged below } \\
\text { invert }\end{array}$ & 0.998 & 0.188 \\
\hline $\mathrm{H}$ & $3 \times$ Glacial & $116 \mathrm{~mm} / \mathrm{yr}$ & & $\begin{array}{l}\text { fractures } \\
\text { plugged below } \\
\text { invert }\end{array}$ & 1.000 & 0.212 \\
\hline$T$ & $\begin{array}{l}\text { Current } \\
\text { Climate }\end{array}$ & $10.14 \mathrm{~mm} / \mathrm{yr}$ & & $\begin{array}{l}\text { fractures } \\
\text { plugged below } \\
\text { EBS }\end{array}$ & 0.984 & 0.180 \\
\hline $\mathrm{J}$ & Monsoon & $24.09 \mathrm{~mm} / \mathrm{yr}$ & & $\begin{array}{l}\text { fractures } \\
\text { plugged below } \\
\text { EBS }\end{array}$ & 1.000 & 0.971 \\
\hline
\end{tabular}

${ }^{*}$ rounded to $3^{\text {rd }}$ decimal place 


\section{CONCLUSIONS}

The EBS successfully drains water entering the EBS for even extreme infiltration rates unless fractures below the EBS become clogged and the permeability of those fractures reduced to matrix permeability values, as might be the result of THC/THM processes or repository construction activities. Drainage performance is impeded if fractures are clogged below the invert but not below the entire EBS, but the EBS can still drain water at significant infiltration rates. Drainage performance is seriously impeded if fractures are clogged below the entire EBS. The EBS is considered to fail with respect to its ability to drain incoming water when the saturation averaged over the bottom half of the invert at the center of the drift reaches a value of 1.

For a "focused glacial" infiltration rate, the EBS successfully drains water entering the EBS. The saturation averaged over the bottom half of the invert never exceeds 0.32 for this infiltration rate, even if the permeability of the backfill and/or the invert is reduced by a factor of 10x. A "focused glacial" infiltration rate is defined as follows: assume a glacial infiltration rate is concentrated spatially such that the entire flux between adjacent pillar centerlines is focused into the intervening drift, and then apply that rate across the entire model domain.

If fractures below the invert become clogged, the EBS fails to drain at an infiltration rate of $3 \mathrm{x}$ the glacial rate. If fractures below the entire EBS become clogged, the EBS fails to drain at an infiltration rate equal to the monsoon infiltration rate.

This water drainage model is based on unsaturated flow through porous media, using an implicit dual permeability (DKM) with active fracture concept (AFC) as represented by the USNT module of the NUFT 3.0s computer code. An effort was made to make this approach as consistent as possible with the approach being used by other AMRs being performed for the EBS PMR and the YMP UZ Site-Scale model. This model is a 2D, steady-state model representative of the center of the repository with waste package heat neglected. The region between the host rock and the backfill is treated as host rock and the region between the invert and the backfill is represented by an impermeable material. 


\section{REFERENCES}

CRWMS M\&O. 1998. Total System Performance Assessment-Viability Assessment Analyses, Technical Basis Document: Chapter 4: Near-Field Geochemical Environment. B0000000001717-4301-00004 REV 01. Las Vegas, Nevada: Civilian Radioactive Waste Management System, Management and Operating Contractor, TRW Environmental Safety Systems, Inc. MOL.19981008.0004.

Nitao, J.J. 1998a. Reference Manual for the NUFT Flow and Transport Code, Version 2.0. UCRL-MA-130651. Livermore, California: Lawrence Livermore National Laboratory.

Nitao, J.J. 1998b. User's Manual for the USNT Module of the NUFT Code, Version 2.0 (NPPhase, NC-Component, Thermal). UCRL-MA-130653. Livermore, California: Lawrence Livermore National Laboratory. 